\title{
On Distributivity and Conditional Distributivity of $S$-uninorms over Uninorms
}

\section{Dragan Jočić}

Novi Sad School of Business, Vladimira Perića-Valtera 4, 21000 Novi Sad, Serbia, dragan.jocic@vps.ns.ac.rs

\section{Ivana Štajner-Papuga}

Department of Mathematics and Informatics, University of Novi Sad, Trg D. Obradovića 4, 21000 Novi Sad, Serbia, ivana.stajner-papuga@dmi.uns.ac.rs

\begin{abstract}
Aggregation operators with an annihilator are in the focus of a significant number of research papers due to their applicability in both theoretical and practical areas of mathematics. Therefore, the main topic of this paper is distributivity and conditional distributivity for some classes of aggregation operators with this property. The characterization of all pairs $(F, G)$ of aggregation operators that are satisfying distributivity law, on both whole and restricted domain, where $F$ is a $S$-uninorm from $U_{\text {min }}$, and $G$ is a t-norm or a uninorm from $U_{\min }$ or $U_{\max }$ is given.
\end{abstract}

Keywords: aggregation operator; annihilator; t-norm; uninorm; S-uninorm; distributivity; conditional distributivity

\section{Introduction}

Lately, aggregation operators have been intensively investigated due to their valuable role in many applications, from mathematics and natural sciences to economics and social sciences (see $[9,11,15])$. Of the special interest is their role in the integration theory [22] and in the utility theory [6, 11, 13]. Regarding this, the main problem that is being studied is the characterizations of the pairs of aggregation operators that are distributive. This issue appeared first in [1]. The more recent results concern t-norms and t-conorms [9], quasi-arithmetic means [2], uninorms and nullnorms [5, 8, 18, 19, 23], semi-t-operators and uninorms [24, $25]$, etc. The issue of the simultaneous distributivity of t-norms and t-conorms over uninorms was investigated in [4]. Also, the problem of distributivity that is directed towards the restricted domain, i.e., the conditional (restricted) 
distributivity, is highly important since this approach can provide more solutions $([12,15,16,17,21,22])$.

The next step is to direct this type of research towards the general commutative aggregation operators with an annihilator, namely towards T-uninorms and Suninorms. The characterization of this type of operators was done in [20]. Therefore, the aim of this paper is to continue the research from [14] where the problem of $T$-uninorms and uninorms was considered. Now the $S$-uninorms, which are a generalization of conjunctive uninorms and nullnorms (t-operators), are observed. The first part of paper considers distributivity of $S$-uninorms over tnorms and t-conorms and uninorms from the class $U_{\min } \cup U_{\max }$. The second part deals with distributivity equations on the restricted domain. Since the conditional distributivity of nullnorms over uninorms was considered in [12], the results given here upgrades the previous results.

\section{Basic Notions}

The core of this research are aggregation operators with an annihilator. As stated in [11], an aggregation operator in $[0,1]^{\mathrm{n}}$ is a function $A^{(\mathrm{n})}:[0,1]^{\mathrm{n}} \rightarrow[0,1]$ that is non-decreasing in each variable and that fulfills the boundary conditions

$$
A^{(\mathrm{n})}(0, \ldots, 0)=0 \quad \text { and } \quad A^{(\mathrm{n})}(1, \ldots, 1)=1 .
$$

The integer $\mathrm{n}$ is the number of input values of the observed aggregation. Further on the binary aggregation operators are being investigated, therefore, the notation $A$ will be used for $A^{(2)}$. Of course, depending on the intended application, some other properties can be required, e.g. associativity, commutativity, idempotency, decomposability, neutral and annihilator elements, etc., (see [11]). Also, if required, the previous can be extended to an arbitrary real interval $[\mathrm{a}, \mathrm{b}]$.

Therefore, the firs part of this section consists of an overview of aggregation operators that are essential for the presented research. Necessary notions concerning distributivity are given in the second part of this section.

\subsection{Uninorms}

The first type of aggregation operators that is needed for the presented research is an aggregation operator with a neutral element, namely the uninorm.

Definition 1 ([27]) A uninorm $U:[0,1]^{2} \rightarrow[0,1]$ is binary aggregation operator that is commutative, associative, and for which there exists a neutral element $e \in[0,1]$, i.e., $U(x, e)=x$ for all $x \in[0,1]$.

If $e=1$, the uninorm $U$ becomes a t-norm (triangular norm) and it is denoted by $T$. If $e=0$, the uninorm $U$ is a t-conorm (triangular conorm) denoted by $S$. 
A uninorm is called conjunctive if $U(0,1)=0$, and disjunctive if $U(0,1)=$ 1. Uninorms for which both functions $U(x, 0)$ and $U(x, 1)$ are continuous, except perhaps at the point $e$, are characterized based on the value $U(0,1)$ by the following theorem from [10].

Theorem 2 ([10]) Let $U$ be a uninorm with a neutral element $e \in(0,1)$ such that both functions $U(x, 1)$ and $U(x, 0)$ are continuous except at the point $x=e$.

If $U(0,1)=0$, then

$$
U(x, y)= \begin{cases}e T\left(\frac{x}{e}, \frac{y}{e}\right) & \text { on }[0, e]^{2}, \\ e+(1-e) S\left(\frac{x-e}{1-e}, \frac{y-e}{1-e}\right) & \text { on }[e, 1]^{2}, \\ \min (x, y) & \text { otherwise, }\end{cases}
$$

where $T$ is a t-norm, and $S$ is a t-conorm.

If $U(0,1)=1$, then

$$
U(x, y)= \begin{cases}e T\left(\frac{x}{e}, \frac{y}{e}\right) & \text { on }[0, e]^{2}, \\ e+(1-e) S\left(\frac{x-e}{1-e}, \frac{y-e}{1-e}\right) & \text { on }[e, 1]^{2}, \\ \max (x, y) & \text { otherwise, }\end{cases}
$$

where $T$ is a t-norm, and $S$ is a t-conorm.

$T$ from (1) (and (2)) is the underlying t-norm of $U$ and $S$ is the underlying tconorm of $U$. The family of all uninorms of the form (1) is denoted by $U_{\min }$, while the family of all uninorms of the form (2) is denoted by $U_{\max }$. More on t-norms, tconorms and uninorms can be found in $[10,11,26,27]$ and the relation between mentioned classes is given by the Figure 1 .

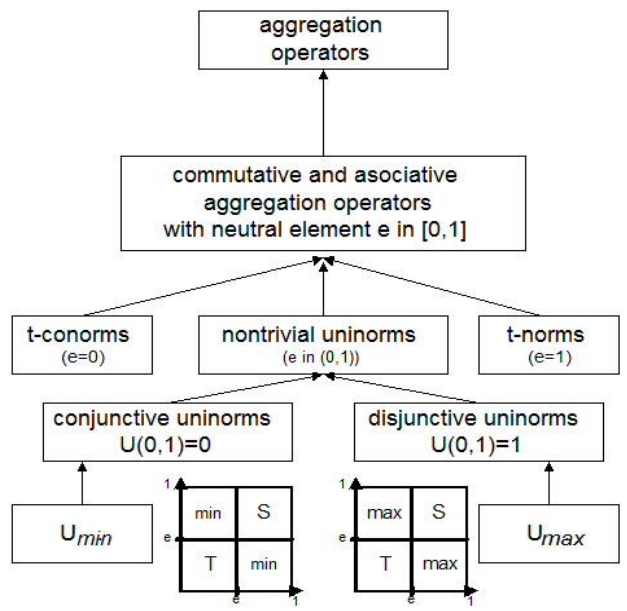

Figure 1

Uninorms 
Example 3 The first uninorms in the terms of Definition 1 were considered by Yager and Rybalov (see [27]) and they are idempotent uninorms $U_{e}^{\min }$ and $U_{e}^{\max }$ from classes $U_{\min }$ and $U_{\max }$, respectively, of the following form

$$
U_{e}^{\min }= \begin{cases}\max & \text { on }[e, 1]^{2}, \\ \min & \text { otherwise, }\end{cases}
$$

and

$$
U_{e}^{\max }= \begin{cases}\min & \text { on }[0, e]^{2}, \\ \max & \text { otherwise } .\end{cases}
$$

Uninorms (3) and (4) are the only idempotent uninorms from classes $U_{\min }$ and $U_{\text {max }}$. On the other hand, the only idempotent t-norm (t-conorm) is minimum (maximum). The idea of a uninorm appeared for the first time in [3] in the form of the aggregative operator, which now can be considered as a generated uninorm.

\subsection{Commutative Aggregation Operators with an Annihilator}

Another type of aggregation operators that is needed for the presented research consists of aggregation operators with an annihilator (absorbing element). An element $a \in[0,1]$ is an annihilator for an aggregation operator $A$ if

$$
A(a, x)=A(x, a)=a
$$

for all $x \in[0,1]$. Further on, the general commutative aggregation operators with an annihilator $a$ are denoted with a-CAOA (see [20]).

For any binary operator $A:[0,1]^{2} \rightarrow[0,1]$ and any element $c \in[0,1]$, the section $A_{c}:[0,1] \rightarrow[0,1]$ is given by

$$
A_{c}(x)=A(c, x) \text {. }
$$

Now, the continuity (discontinuity) of sections $A_{0}$ and $A_{1}$ plays a crucial role in classification and characterization of associative a-CAOA operators as given by Figure 2 (see [20]).

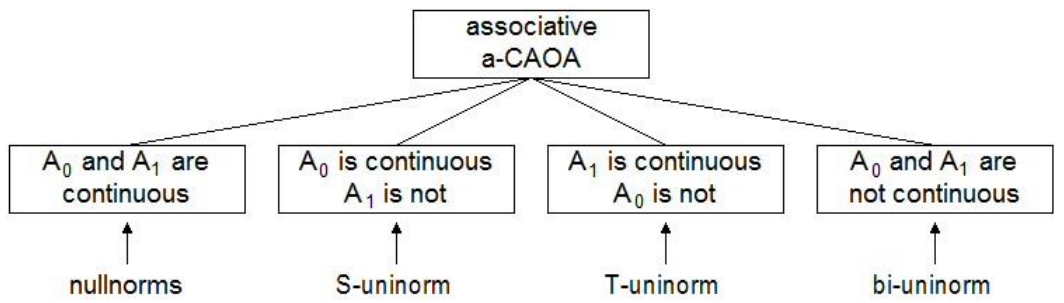

Figure 2

Classification of a-CAOA 


\subsection{1 $S$-uninorms}

Definition 5 ([20]) A binary operator $A:[0,1]^{2} \rightarrow[0,1]$ is called a $S$-uninorm if it is an associative a-CAOA satisfying the following properties:

- Section $A_{0}$ is continuous and section $A_{1}$ is not.

- $\quad$ There is $e \in(0,1)$ such that $e$ is an idempotent element, the section $A_{e}$ is continuous and $A_{e}(1)=1$.

Theorem 6 ([20]) Let $A:[0,1]^{2} \rightarrow[0,1]$ be a binary operator. The following statements are equivalent:

- A is a $S$-uninorm.

- $\quad$ There exists $a \in[0,1)$, a $t$-conorm $S^{\prime}$ and a conjunctive uninorm $U^{\prime}$ with neutral element $e^{\prime} \in(0,1)$ such that $A$ is given by

$$
\begin{cases}a S^{\prime}\left(\frac{x}{a}, \frac{y}{a}\right), & A(x, y)= \\ a+(1-a) U^{\prime}\left(\frac{x-a}{1-a}, \frac{y-a}{1-a}\right), & \text { on }[0, a]^{2}, \\ a, & \text { on }[a, 1]^{2}, \\ \text { on }[0, a] \times[a, 1] \cup[a, 1] \times[0, a] .\end{cases}
$$

- $\quad$ There exists $a \in[0,1)$, a t-conorm $S$ and a conjunctive uninorm $U$ with neutral element $e \in(0,1)$ such that $U(x, a) \leq a$ for all $x \in[0,1], U \leq S$ and $A=\operatorname{med}(a, U, S)$.

Remark 7 Let $A:[0,1]^{2} \rightarrow[0,1]$ be a $S$-uninorm.

- $\quad$ For a $=0$,operator $A$ becomes a conjunctive uninorm, i.e., $A=U^{\prime}$.

- If a $\neq 1$, in order to ensure the discontinuity of $A_{1}$ and since $A_{e}(1)=1$, $\mathrm{a}<\mathrm{e}$.

- If $U^{\prime} \in U_{\text {min }}$, then $A$ is a $S$-uninorm from $U_{\text {min }}$.

Example 8 Binary operator $A:[0,1]^{2} \rightarrow[0,1]$ of the form

$$
A(x, y)= \begin{cases}a, & \text { on }[0, a] \times[a, 1] \cup[a, 1] \times[0, a], \\ \max (x, y), & \text { on }[0, a]^{2} \cup[e, 1]^{2}, \\ \min (x, y), & \text { otherwise, }\end{cases}
$$

is an idempotent $S$-uninorm from $U_{\min }$ with annihilator $a$. It is obtained from (5) when for the t-conorm $S^{\prime}$ the operator max is taken and the uninorm $U^{\prime}$ is $U_{e}^{\min }$.

\subsection{Distributivity on the Whole Domain}

Let $A, B:[0,1]^{2} \rightarrow[0,1]$ be two arbitrary operators.

- $A$ is left distributive over $B$ if

$$
A(x, B(y, z))=B(A(x, y), A(x, z)), \quad \text { for all } x, y, z \in[0,1] \text {. }
$$

- $A$ is right distributive over $\mathrm{B}$ if

$$
A(B(y, z), x)=B(A(y, x), A(z, x)), \quad \text { for all } x, y, z \in[0,1] \text {. }
$$


The previous two functional equations are called the left and the right distributivity laws (see [1], p. 318), and are denoted with (LD) and (RD). Of course, for a commutative $A$, (LD) and (RD) coincide. Now, $A$ is distributive over $B$ if it is both left and right distributive over $B$.

The following two lemmas answer some starting questions regarding distributivity.

Lemma 9 ([5]) Let $X \neq \emptyset, A: X^{2} \rightarrow X$ and let $e \in Y$, where $Y \subset X$, be a neutral element for the operator $A$ on $Y\left(\forall_{x \in Y} A(e, x)=A(x, e)=x\right)$. If the operator $A$ is left or right distributive over some operator $B: X^{2} \rightarrow X$ that fulfils $B(e, e)=e$, then $B$ is idempotent on $Y$.

Lemma 10 ([5]) All increasing functions are distributive over max and min.

\subsection{Distributivity on the Restricted Domain}

As seen in [9], the problem of distributivity of a t-norm Tover a t-conorm $S$ on the whole domain has only the trivial solution, i.e., t-conorm in question has to be $S_{M}=$ max. In order to obtain more solutions, the domain had to be restrict. That is, for the classical functional equation $T(x, S(y, z))=S(T(x, y), T(x, z))$, the additional condition $S(y, z)<1$ is necessary (see [15], p. 138). This distributivity under the given restriction is called the conditional (restricted) distributivity and it is denoted with (CD).

The similar restriction holds for conditional distributivity of a t-conorm $S$ over a tnorm $T$ :

(CD) $S(x, T(y, z))=T(S(x, y), S(x, z))$, whenever $T(y, z)>0$, for all $x, y, z \in[0,1]$.

The previous concept can be extended to some more general aggregation operators, as given by the following definition.

Definition 11 Let $F$ be a $S$-uninorm with annihilator $a \in(0,1)$ and let $G$ be a $t$ norm or $G \in U_{\min } \cup U_{\max } . F$ is conditionally distributive $(C D)$ over $G$ if for all $x, y, z \in[0,1]$ the following holds

(CD) $\quad F(x, G(y, z))=G(F(x, y), F(x, z))$, whenever $G(y, z)>0$.

Lemma 12 ([5]) All increasing functions are conditionally distributive over max and $\mathrm{min}$. 


\section{Distributivity Laws for $S$-uninorms over Uninorms}

This section considers distributivity of a $S$-uninorm from $U_{\min }$ with an annihilator $a$ over a t-conorm, a t-norm or a uninorm from $U_{\min } \cup U_{\max }$.

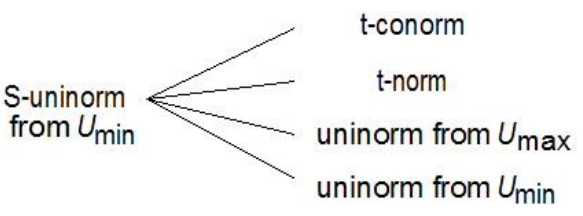

Figure 3

Topics of the Section 3

Since a $S$-uninorm is a commutative operator, there is no need to discus (LD) and (RD) separately. The distributivity of $F$ over $G$ for $a=0$, that is for $F$ being a uninorm from $U_{\min }$, was investigated in $[18,19]$. Therefore, the further assumption is that $a \in(0,1)$. Also, further on the neutral element of the underlying uninorm of $F$ will be denote by $e$. The similar issues were simultaneously investigated in [7] and some of the following results are independently confirmed.

\section{1 $S$-uninorm vs. t-norm and t-conorm}

The results concerning t-norms and t-conorms are not very surprising since the idempotence still plays an important role. Additionally, some aspects of the proofs are analogous to ones from [14], therefore, they can be omitted. Also, see [7].

Theorem 13 Let $F$ be a $S$-uninorm from $U_{\min }$ and let $T$ be a $t$-norm. $F$ is distributive over $T$ if and only if $T=\min$.

Theorem 14 Let $F$ be a $S$-uninorm in $U_{\min }$ and let $S$ be a t-conorm.

(i) If $F$ is distributive over $S$ then $S(x, x)=x$ for all $x<e$.

(ii) Let the function $s(x)=S(x, x)$ be left-continuous at the point $x=e$. Then, $F$ is distributive over $S$ if and only if $S=\max$.

\section{2 $S$-uninorm vs. uninorm from $U_{\min }$}

Now the second operator is a conjunctive uninorm $U$ with continuous functions (except perhaps at the point $e) U(x, 0)$ and $U(x, 1)$. The first two lemmas are necessary for the proof of the main theorem of this subsection.

The main idea behind the proofs that follow is analogous to one from [14] for $F$ being a $T$-uninorm from $U_{\max }$ and $U$ be a uninorm from the class $U_{\max }$. However, it is very interesting to see how the duality of operators influences the process of proving and, therefore, the proofs in this section are not omitted. 
Lemma 15 Let $F$ be a $S$-uninorm from $U_{\min }$ and let $U$ be a uninorm from the class $U_{\min }$ with a neutral element $e_{1} \in(0,1)$. If $F$ is distributive over $U$ then $e_{1}>a$.

Proof. Let suppose the opposite, i.e., $e_{1}<a$. For $x=e_{1}, y=0, z=1$ assumed distributivity gives the following contradiction

$$
e_{1}=F\left(e_{1}, 0\right)=F\left(e_{1}, U(0,1)\right)=U\left(F\left(e_{1}, 0\right), F\left(e_{1}, 1\right)\right)=U\left(e_{1}, a\right)=a .
$$

Therefore, $e_{1} \geq a$.

If the assumption is now $e_{1}=a$, then for $e_{1}=a<x<e$ and $y=0, z=1$, from the distributivity law follows

$$
a=F(x, 0)=F(x, U(0,1))=U(F(x, 0), F(x, 1))=U(a, x)=U\left(e_{1}, x\right)=x .
$$

That is again a contradiction and, hence, $e_{1}>a$.

The previous lemma shows that $e_{1}>a$. The following one will explain relation between neutral elements $e$ and $e_{1}$. Element $e$, as stated at the beginning of this section, is the neutral element of of the underlying uninorm of the observed $S$ uninorm, while element $e_{1}$ is the neutral element of the considered uninorm $U$ from $U_{\min }$.

Lemma 16 Let $F$ be a $S$-uninorm from $U_{\min }$ and let $U$ be a uninorm from the class $U_{\text {min }}$ with a neutral element $e_{1} \in(0,1)$. If $F$ is distributive over $U$ then $e_{1}=e$ or $e_{1}<e$.

Proof. Let suppose the opposite, that is that $e_{1}>e$. For $e_{1}<x<1, y=e, z=$ 1 , the assumed distributivity leads to the following contradiction

$$
x=F(x, e)=F(x, U(e, 1))=U(F(x, e), F(x, 1))=U(x, 1)=1 .
$$

Therefore, either $e_{1}=e$ or $e_{1}<e$ holds

The following theorem is the main result of this subsection.

Theorem 17 Let $F$ be a $S$-uninorm from $U_{\min }$ and $U$ be a uninorm from the class $U_{\min }$ with a neutral element $e_{1} \in(0,1)$ and underlying $t$-conorm $S$ such that $S(x, x)$ is left-continuous at the point $x=e . F$ is distributive over $U$ if and only if $e_{1}>a$ and exactly one of the following cases is fulfilled:

(i) $e_{1}=e$, and $U$ is an idempotent uninorm, i.e., $U=U_{e_{1}}^{\min }$,

(ii) $e_{1}<e, U=U_{e_{1}}^{\min }$, and $F$ is given by

$$
F(\mathrm{x}, \mathrm{y})=
$$




$$
\begin{cases}a S^{\prime}\left(\frac{x}{a}, \frac{y}{a}\right) & \text { on }[0, a]^{2}, \\ a+\left(e_{1}-a\right) T_{1}^{\prime}\left(\frac{x-a}{e_{1}-a}, \frac{y-a}{e_{1}-a}\right) & \text { on }\left[a, e_{1}\right]^{2}, \\ e_{1}+\left(e-e_{1}\right) T_{1}^{\prime \prime}\left(\frac{x-e_{1}}{e-e_{1}}, \frac{y-e_{1}}{e-e_{1}}\right) & \text { on }\left[e_{1}, e\right]^{2}, \\ e+(1-e) S_{1}\left(\frac{x-e}{1-e}, \frac{y-e}{1-e}\right) & \text { on }[e, 1]^{2}, \\ a & \text { on }[0, a] \times[a, 1] \cup[a, 1] \times[0, a], \\ \min (\mathrm{x}, \mathrm{y}) & \text { otherwise, }\end{cases}
$$

where $S_{1}$ and $S^{\prime}$ are $t$-conorms, and $T_{1}{ }^{\prime}$ and $T_{1}{ }^{\prime \prime}$ are $t$-norms.

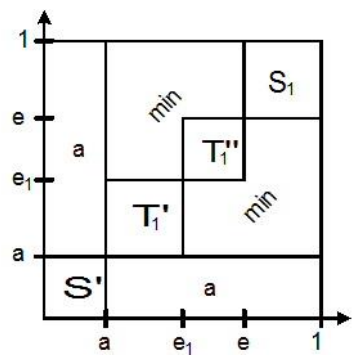

Figure 4

The form of the S-uninorm from Theorem 17

Proof. $(\Rightarrow)$ Let $F$ be a $S$-uninorm from the class $U_{\min }$ and let $U$ be a uninorm from $U_{\text {min }}$ that satisfy distributivity law. From Lemma 15 and Lemma 16 follows that $e_{1}>a$, and either $e=e_{1}$ or $e>e_{1}$. The next step is to prove that $U$ is an idempotent uninorm and it can be done analogously to the corresponding proof from [14]. Consequently, $U$ is an idempotent uninorm, i.e., $U=U_{e_{1}}^{\min }$, and the claim (i) holds.

The next issue is the structure of $F$ for $e>e_{1}$. The first step is to show that $F\left(e_{1}, e_{1}\right)=e_{1}$. Let $x=y=e_{1}, z=e$. From the assumed distributivity follows $e_{1}=F\left(e_{1}, e\right)=F\left(e_{1}, U\left(e_{1}, e\right)\right)=U\left(F\left(e_{1}, e_{1}\right), F\left(e_{1}, e\right)\right)=U\left(F\left(e_{1}, e_{1}\right), e_{1}\right)=$ $F\left(e_{1}, e_{1}\right)$.

For $a \leq x \leq e_{1}$, due to the distributivity law, holds

$$
x=F(x, e)=F\left(x, U\left(e_{1}, e\right)\right)=U\left(F\left(x, e_{1}\right), F(x, e)\right)=U\left(F\left(x, e_{1}\right), x\right) .
$$

Since $F\left(x, e_{1}\right) \leq F(x, e)=x \leq e_{1}$ and $U=U_{e_{1}}^{\min }$, the following can be obtained

$$
x=U\left(F\left(x, e_{1}\right), x\right)=\min \left(F\left(x, e_{1}\right), x\right)=F\left(x, e_{1}\right) .
$$

Also, for $e_{1} \leq x \leq e$ holds $e_{1}=F\left(e, e_{1}\right) \geq F\left(x, e_{1}\right) \geq F\left(e_{1}, e_{1}\right)=e_{1}$. Therefore,

$$
F\left(x, e_{1}\right)= \begin{cases}x & \text { for } a \leq x \leq e_{1}, \\ e_{1} & \text { for } e_{1} \leq x \leq e .\end{cases}
$$


Now, from (5) and (8) follows that $F$ has to be of the form (7).

$(\Leftarrow)$ It is enough to prove the claim (ii), since the proof for the claim (i) is analogous. Therefore, let $F$ be a $S$-uninorm given by (7) and $U=U_{e_{1}}^{\min }$. To prove the distributivity law, we have to consider $4^{3}=64$ cases. However, directly from the Lemma 10, distributivity for $x \in[0,1]$ and $(y, z) \in\left[0, e_{1}\right]^{2} \cup\left[e_{1}, 1\right]^{2}$ holds. Otherwise, for $y<e_{1}<z, U(y, z)=y$ and $F(x, y) \leq F(x, z)$. Now, $L$ will be used to denote the left side of distributivity law, i.e., $L=F(x, U(y, z))=F(x, y)$. Also, the right side is denoted with $R$, i.e., $R=U(F(x, y), F(x, z))$. As in [14], there are four cases for evaluation of the $R: x \geq e, e_{1} \leq x \leq e, a \leq x \leq e_{1}$ and $x \leq a$. In all cases $R=\min (F(x, y), F(x, z))=F(x, y)$ is obtained.

As seen above, in all considered cases $L=R$ is obtained, which proves that the distributivity law holds.

Remark 18 a) If the assumption of the of left-continuity for the function $S(x, x)$ at $x=e$ is omitted, the claim (i) from the previous theorem still holds, while the claim (ii), according to Theorem 14, is of the following form: If $F$ is distributive over $U$ then $U(x, x)=$ for $x<e$ and $F$ is given by (7).

b) The restriction of the previous theorem to $a=0$, i.e., to $S$-uninorm being just a uninorm from the class $U_{\min }$, has been shown in $[18,19]$. The case (i) generalizes the Proposition 6.6 from $[18,19]$, and the case (ii) generalizes the Proposition 6.7 from [18].

\section{3 $S$-uninorm vs. Uninorm from $U_{\max }$}

The second operator in this subsection is a disjunctive uninorm $U$ with continuous functions (except perhaps at the point $e) U(x, 0)$ and $U(x, 1)$, i.e., a uninorm from the class $U_{\max }$. Now, as in [14], the following can be shown. Also, see [7].

Lemma 19 Let $F$ be a $S$-uninorm in $U_{\text {min }}$ and let $U$ be a uninorm from the class $U_{\text {max }}$ with a neutral element $e_{1} \in(0,1)$. If $F$ is distributive over $U$ then $e_{1}<a$.

Theorem 20 Let $F$ be a $S$-uninorm in $U_{\text {min }}$ and let $U$ be a uninorm from the class $U_{\max }$ with a neutral element $e_{1} \in(0,1)$ and underlying t-conorm $S$ such that $S(x, x)$ is left-continuous at the point $x=e . F$ is distributive over $U$ if and only if $e_{1}<a, U=U_{e_{1}}^{\max }$ and $F$ is given by

$$
F(\mathrm{x}, \mathrm{y})=
$$




$$
\begin{cases}e_{1} S_{1}{ }^{\prime}\left(\frac{x}{e_{1}}, \frac{y}{e_{1}}\right) & \text { on }\left[0, e_{1}\right]^{2}, \\ e_{1}+\left(a-e_{1}\right) S_{2}{ }^{\prime}\left(\frac{x-e_{1}}{a-e_{1}}, \frac{y-e_{1}}{a-e_{1}}\right) & \text { on }\left[e_{1}, a\right]^{2}, \\ a+(1-a) U^{\prime}\left(\frac{x-a}{1-a}, \frac{y-a}{1-a}\right) & \text { on }[a, 1]^{2}, \\ a & \text { on }[0, a] \times[a, 1] \cup[a, 1] \times[0, a], \\ \max (\mathrm{x}, \mathrm{y}) & \text { otherwise, }\end{cases}
$$

where $S_{1}{ }^{\prime}, S_{2}{ }^{\prime}$ are $t$-conorms, and $U^{\prime}$ is a uninorm from the class $U_{\min }$.

Remark 21 According to [18] (see Lemma 6.5), if $F$ is a uninorm from the class $U_{\text {min }}$, i.e., a $S$-uninorm in $U_{\min }$ with annihilator a $=0$, then there is no uninorm $U$ from the class $U_{\max }$ such that $F$ is distributive over $U$. Theorem 20 shows that, when $S$-uninorm in $U_{\min }$ has annihilator a $\in(0,1)$, there is a uninorm $U=$ $U_{e_{1}}^{\max } \in U_{\max }$ with neutral element $\mathrm{e}_{1}<a$ such that $F$ is distributive over $U$.

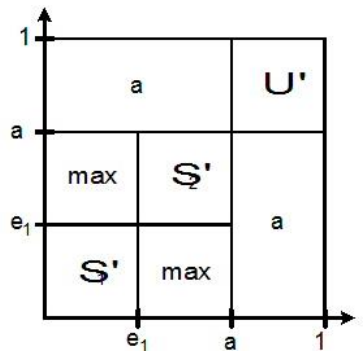

Figure 5

The form of the S-uninorm from Theorem 20

\section{Distributivity Laws on Restricted Domain for $S$ - Uninorms over Uninorms}

Theorems from the previous section illustrate that the distributivity law (on whole domain) is a very strong condition since it considerably simplifies the structure of the inner operator. In this case, the inner operator is reduced to an idempotent operator. The research so farhas shown that restriction of the domain of the distributivity law can provide some new solutions that are non-idempotent. Therefore, this section contains the counterparts of theorems 13, 17, 20 from the previous section, now done for the restricted domain. Now, in order to characterize all pairs $(F, G)$ satisfying (CD) condition, some kind of continuity for $F$ and $G$ has to hold (see [15]). The following results are counterparts to results from [14] and, for the sake of rounding up this topic, the proofs are not omitted. 


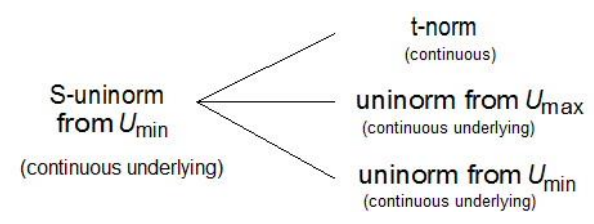

Figure 6

Topics of the Section 4

\section{1 $S$-uninorm vs. t-norm}

Theorem 22 Let $F$ be a $S$-uninorm in $U_{\min }$ with continuous underlying $t$-conorm $S^{\prime}$, and let $T$ be a continuous t-norm. $F$ is conditionally distributive over $T$ if and only if exactly one of the following cases is fulfilled:

(i) $T=T_{M}$;

(ii) there is $c \in(0, a]$ such that $T$ is given by

$$
T(x, y)= \begin{cases}c T_{L}\left(\frac{x}{c}, \frac{y}{c}\right) & \text { on }[0, c]^{2}, \\ \min (x, y) & \text { otherwise, }\end{cases}
$$

and $F$ is given by

$$
\begin{cases}c S_{P}\left(\frac{x}{c}, \frac{y}{c}\right) & \text { on }[0, c]^{2}, \\ c+(a-c) S_{1}\left(\frac{x-c}{a-c}, \frac{y-c}{a-c}\right) & \text { on }[c, a]^{2}, \\ a+(1-a) U^{\prime}\left(\frac{x-a}{1-a}, \frac{y-a}{1-a}\right) & \text { on }[a, 1]^{2}, \\ a & \text { on }[0, a] \times[a, 1] \cup[a, 1] \times[0, a], \\ \max & \text { otherwise, }\end{cases}
$$

where $S_{1}$ is a continuous $t$-conorm, and $U^{\prime}$ is a uninorm from the class $U_{\min }$.
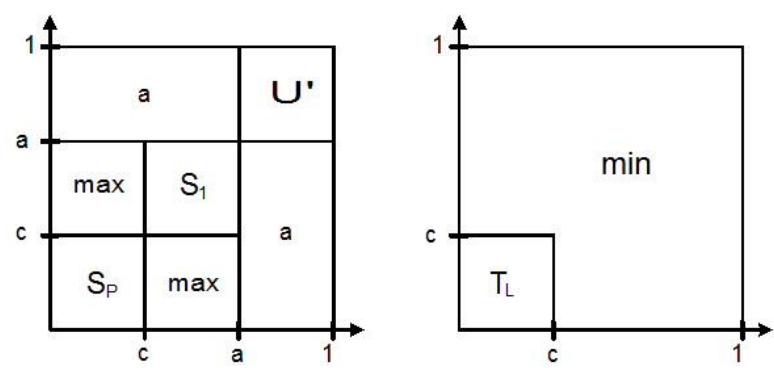

Figure 7

Conditionally distributive pair from Theorem 22 
Proof. $(\Rightarrow)$ Let $F$ be conditionally distributive over $T$.

For $x \geq a$, as in Theorem 13, it can be shown that $T(x, x)=x$.

Let $x \leq a$. If $c \in(0, a]$ is an idempotent element of $T$, then, as in [14], there can be shown that all elements from $[c, a]$ are idempotents of $T$. Hence, either all elements from $[0,1]$ are idempotent elements for t-norm $T$ and, therefore $T=$ $T_{M}=$ min, or there is the smallest nontrivial idempotent element $c \in(0, a]$ of $T$, i.e.,

$$
T(x, y)= \begin{cases}c T^{*}\left(\frac{x}{c}, \frac{c}{c}\right) & \text { on }[0, c]^{2}, \\ \min (x, y) & \text { otherwise, }\end{cases}
$$

where $T^{*}$ is a continuous Archimedean t-norm. Now, as in Theorem 5.21 from [15] (pp. 138-140), it can be proved that $c$ is also an idempotent element of $F$, i.e., $S$-uninorm $F$ on the square $[0, a]^{2}$ is of the following form

$$
F(x, y)= \begin{cases}c S_{2}\left(\frac{x}{c}, \frac{y}{c}\right) & \text { if }(x, y) \in[0, c]^{2}, \\ c+(a-c) S_{1}\left(\frac{x-c}{a-c}, \frac{y-c}{a-c}\right) & \text { if }(x, y) \in[c, a]^{2}, \\ \max (x, y) & \text { otherwise, }\end{cases}
$$

where $S_{1}$ and $S_{2}$ are continuous t-conorms. Also, in the same manner as in Theorem 5.21 from [15] (pp. 138-140), it can be obtained that $T^{*}$ is a nilpotent tnorm, i.e., $T$ is of the form (11), and that $S_{2}$ is a strict t-conorm such that $F$ is of the form (12).

$(\Leftarrow)$ Now, if the starting assumption is that $T$ is a t-norm of the form (11) and $F$ a $S$-uninorm of the form (12), it can be easily shown that condition (CD) holds. For input values from $[0, c]^{2}$ the problem is reduced to the pair $\left(S_{P}, T_{L}\right)$ which satisfies (CD), and in all other cases it follows from Lemma 12.

Example 23 Operator F given by

$$
\begin{gathered}
F(x, y)= \\
\begin{cases}\max & \text { if } \quad(x, y) \in\left[\frac{3}{5}, 1\right]^{2} \cup\left[\frac{1}{4}, \frac{1}{2}\right] \times\left[0, \frac{1}{2}\right] \cup\left[0, \frac{1}{2}\right] \times\left[\frac{1}{4}, \frac{1}{2}\right], \\
x+y-4 x y & \text { if } \quad(x, y) \in\left[0, \frac{1}{4}\right]^{2}, \\
\frac{1}{2} & \text { if } \quad(x, y) \in\left[0, \frac{1}{2}\right] \times\left[\frac{1}{2}, 1\right] \cup\left[\frac{1}{2}, 1\right] \times\left[0, \frac{1}{2}\right], \\
\min & \text { otherwise, }\end{cases}
\end{gathered}
$$

is a $S$-uninorm in $U_{\min }$ with annihilator $a=\frac{1}{2}$, obtained by (12) where $U^{\prime}=U_{\frac{3}{5}}^{\min }$, $S_{1}=\max$ and $c=\frac{1}{4}$. The corresponding t-norm is of the form (11). 


\section{2 $S$-uninorm vs. Uninorm from $U_{\text {min }}$}

Theorem 24 Let $F$ be a $S$-uninorm in $U_{\min }$ with a continuous underlying $t$ conorm $S^{\prime}$, and let $U$ be a uninorm from the class $U_{\text {min }}$ with a neutral element $e_{1} \in(0,1)$ and continuous underlying $t$-norm and $t$-conorm. $F$ is conditionally distributive over $U$ if and only if $e_{1}>a$ and exactly one of the following cases is fulfilled:

(i) $e_{1}=e$, and $U$ is an idempotent uninorm, i.e., $U=U_{e_{1}}^{\min }$,

(ii) $e_{1}=e$, and there is a $c \in(0, a]$ such that $F$ and $U$ are given by

$$
U(x, y)= \begin{cases}\max (x, y) & \text { on }\left[e_{1}, 1\right]^{2}, \\ c T_{L}\left(\frac{x}{c}, \frac{y}{c}\right) & \text { on }[0, c]^{2}, \\ \min (x, y) & \text { otherwise }\end{cases}
$$

and

$$
\begin{cases}c S_{P}\left(\frac{x}{c}, \frac{y}{c}\right) & \text { on }[0, c]^{2}, \\ c+(a-c) S_{2}\left(\frac{x-c}{a-c}, \frac{y-c}{a-c}\right) & \text { on }[c, a]^{2}, \\ a+(1-a) U^{\prime}\left(\frac{x-a}{1-a}, \frac{y-a}{1-a}\right) & \text { on }[a, 1]^{2}, \\ a & \text { on }[0, a] \times[a, 1] \cup[a, 1] \times[0, a], \\ \max & \text { otherwise, }\end{cases}
$$

(iii) $e_{1}<e, U=U_{e_{1}}^{\min }$, and $F$ is given by (7),

(iv) $e_{1}<e$, and there is a $c \in(0, a]$ such that $U$ is given by (13) and $F$ is given by

$$
\begin{cases}c S_{P}\left(\frac{x}{c}, \frac{y}{c}\right) & \text { on }[0, c]^{2}, \\ c+(a-c) S_{3}\left(\frac{x-c}{a-c}, \frac{y-c}{a-c}\right) & \text { on }[c, a]^{2}, \\ a+\left(e_{1}-a\right) T_{1}{ }^{\prime}\left(\frac{x-a}{e_{1}-a}, \frac{y-a}{e_{1}-a}\right) & \text { on }\left[a, e_{1}\right]^{2}, \\ e_{1}+\left(e-e_{1}\right) T_{1}^{\prime \prime}\left(\frac{x-e_{1}}{e-e_{1}}, \frac{y-e_{1}}{e-e_{1}}\right) & \text { on }\left[e_{1}, e\right]^{2}, \\ e+(1-e) S_{1}\left(\frac{x-e}{1-e}, \frac{y-e}{1-e}\right) & \text { on }[e, 1]^{2}, \\ \max & \text { on }[c, a] \times[0, c] \cup[0, c] \times[c, a], \\ a & \text { on }[0, a] \times[a, 1] \cup[a, 1] \times[0, a], \\ \min & \text { otherwise, }\end{cases}
$$

where $U^{\prime}$ is a uninorm from the class $U_{\text {min }}, T_{1}{ }^{\prime}, T_{1}{ }^{\prime \prime}$ are $t$-norms, $S_{1}$ is a $t$ conorm, and $S_{2}, S_{3}$ are continuous t-conorms. 


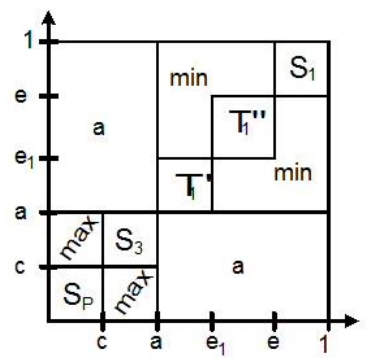

Figure 8

Operator $F$ from Theorem 24 (iv)

Proof. $(\Rightarrow)$ Let $F$ be conditionally distributive over $U$. The first step is to prove that $e_{1}>a$, which can be done by supposing the opposite (see [14]).

As in Lemma 16, it can be proved that either $e=e_{1}$ or $e>e_{1}$ holds. In the sequel it is supposed that $e>e_{1}$, since the case $e=e_{1}$ is similar.

For $x \geq a$, as in Theorem 17, holds $U(x, x)=x$ and the structure of $F$ on the square $[a, 1]^{2}$ is given as in (15).

For $x \leq a$, as in Theorem 22, it can be proved that either $U$ is an idempotent uninorm and $F$ is given by (7), or there is a $c \in(0, a]$ such that $U$ and $F$ are given by (13) and (15), respectively.

$(\Leftarrow)$ On the other hand, if the observed $S$-uninorm $F$ and uninorm $U$ are of forms (15) and (13), the (CD) condition can be proved as in Theorem 17

\section{3 $S$-uninorm vs. Uninorm from $U_{\max }$}

Theorem 25 Let $F$ be a $S$-uninorm in $U_{\min }$ with a continuous underlying $t$ conorm $S^{\prime}$, and let $U$ be a uninorm from the class $U_{\max }$ with a neutral element $e_{1} \in(0,1)$ and continuous underlying $t$-norm and $t$-conorm. $F$ is conditionally distributive over $U$ if and only if $e_{1}<a$ and exactly one of the following cases is fulfilled:

(i) $U=U_{e_{1}}^{\max }$, and $F$ is given by (9);

(ii) there is a $c \in\left(0, e_{1}\right]$ such that $F$ and $U$ are given by

$$
U(x, y)= \begin{cases}\max (x, y) & \text { on }\left(e_{1}, 1\right] \times[0,1] \cup[0,1] \times\left(e_{1}, 1\right] \\ c T_{L}\left(\frac{x}{c}, \frac{y}{c}\right) & \text { on }[0, c]^{2} \\ \min (x, y) & \text { otherwise }\end{cases}
$$

and

$$
F(x, y)=
$$




$$
\begin{cases}c S_{P}\left(\frac{x}{c}, \frac{y}{c}\right) & \text { on }[0, c]^{2}, \\ c+\left(e_{1}-c\right) S_{2}{ }^{\prime \prime}\left(\frac{x-c}{e_{1}-c}, \frac{y-c}{e_{1}-c}\right) & \text { on }\left[c, e_{1}\right]^{2}, \\ e_{1}+\left(a-e_{1}\right) S_{2}{ }^{\prime}\left(\frac{x-e_{1}}{a-e_{1}}, \frac{y-e_{1}}{a-e_{1}}\right) & \text { on }\left[e_{1}, a\right]^{2}, \\ a+(1-a) U^{\prime}\left(\frac{x-a}{1-a}, \frac{y-a}{1-a}\right) & \text { on }[a, 1]^{2}, \\ a & \text { on }[0, a] \times[a, 1] \cup[a, 1] \times[0, a], \\ \max & \text { otherwise, }\end{cases}
$$

where $U^{\prime}$ is a uninorm from the class $U_{\text {min }}$, and $S_{2}{ }^{\prime}, S_{2}{ }^{\prime \prime}$ are continuous t-conorms.
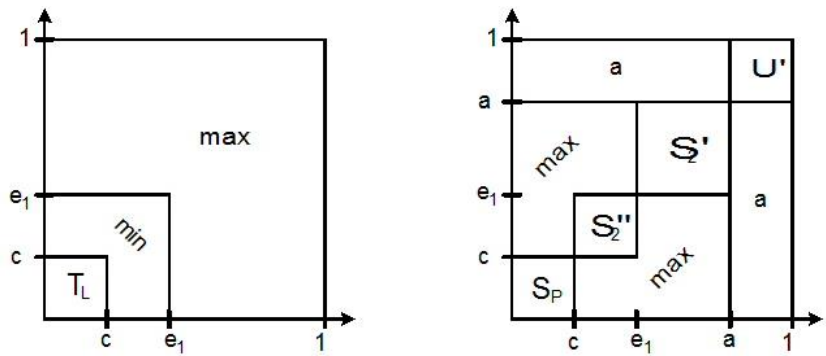

Figure 9

Conditionally distributive pair from Theorem 25 (ii)

Proof. $(\Rightarrow)$ Let $F$ be conditionally distributive over $U$.

As in Theorem 20, it can be shown that $e_{1}<a$ and that, for $x \geq a$, holds $U(x, x)=x$

Now, analogously to [14], it can be shown that $U=\max$ on the square $\left[e_{1}, 1\right]^{2}$.

The nex step is to show that $e_{1}$ is an idempotent element of $F$. For $x=e_{1}, z=e_{1}$, and an arbitrary $y \in\left(0, e_{1}\right)$ from equation $(\mathrm{CD})$ follows

$$
F\left(e_{1}, y\right)=F\left(e_{1}, U\left(y, e_{1}\right)\right)=U\left(F\left(e_{1}, y\right), F\left(e_{1}, e_{1}\right)\right) .
$$

Due to the assumption of continuity, the previous equality can be extended to $y=0$ and $e_{1}=F\left(e_{1}, 0\right)=U\left(e_{1}, F\left(e_{1}, e_{1}\right)\right)=F\left(e_{1}, e_{1}\right)$. Now, since $S^{\prime}=\left.F\right|_{[0, a]^{2}}$ is a continuous t-conorm immediately follows that $S^{\prime}$ is ordinal sum $S_{1}{ }^{\prime}$ and $S_{2}{ }^{\prime}$, i.e., $F$ is given by (9). Therefore, $U=\max$ on the square $\left[e_{1}, 1\right]^{2}$ and $F$ is given by (9).

For $x \leq e_{1}$, analogous to Theorem 22 for $x \leq a$, it can be proved that either $U$ is an idempotent uninorm and $F$ is given by (9), or there is a $c \in\left(0, e_{1}\right]$ such that $U, F$ are given by (17) and (18) respectively.

$(\Leftarrow)$ On the other hand, if the observed $S$-uninorm $F$ and uninorm $U$ are of forms (18) and (17), respectively, the (CD) condition can be shown as in Theorem 17. 


\section{Conclusion}

Investigation of distributivity and conditional distributivity of a $S$-uninorm from $U_{\text {min }}$ with an annihilator $a \in(0,1)$ is presented in this paper. The first set of results given in the third section concerns distributivity law on the whole domain and they extend and upgrade the corresponding ones from [18, 19]. Section 4 illustrates that the conditional distributivity produces a larger variety of solutions and the represented research is the continuation of investigations of conditional distributivity for aggregation operators with annihilator from $[12,17]$. The further research will be directed towards possible application of the obtained structures to utility theory.

\section{Acknowledgement}

This work was supported by the Ministry of Education, Science and Technological Development of Republic of Serbia 174009.

\section{References}

[1] J. Aczél, Lectures on Functional Equations and their Applications, Academic Press, New York 1966

[2] T. Calvo, On some solutions of the distributivity equations, Fuzzy Sets and Systems 104 (1999) 85-96

[3] J. Dombi, Basic concepts for a theory of evaluation: The aggregative operator, European Journal of Operational Research 10 (1982) 282-293

[4] J. Dombi, On a certain class of aggregative operators, Information Sciences 245 (2013) 313-328

[5] J. Drewniak, P. Drygas, E. Rak, Distributivity between uninorms and nullnorms, Fuzzy Sets and Systems 159 (2008) 1646-1657

[6] D. Dubois, E. Pap, H. Prade, Hybrid probabilictic-possibilistic mixtures and utility functions, Preferences and Decisions under Incomplete Knowledge, Studies in Fuzziness and Soft Computing, Vol. 51, Springer-Verlag 2000, $51-73$

[7] B. W. Fang, B. Q, Hu, Distributivity and conditional distributivity for $S$ uninorms, Fuzzy Sets and Systems, corrected proof

[8] Q. Feng, Z. Bin, The distributive equations for idempotent uninorms and nullnorms, Fuzzy Sets and Systems 155 (2005) 446-458

[9] J. C. Fodor, M. Roubens, Fuzzy Preference Modelling and Multicriteria Decision Support, Kluwer Academic Publishers, Dordrecht, 1994

[10] J. C. Fodor, R. R. Yager, A. Rybalov, Structure of uninorms, Internat. J. Uncertainty, Fuzziness and Knowledge-Based Systems 5 (1997) 411-427

[11] M. Grabisch, J. Marichal, R. Mesiar, E. Pap, Aggregations Functions, Cambridge University Press, 2009 
[12] D. Jočić, I. Štajner-Papuga, Restricted distributivity for aggregation operators with absorbing element, Fuzzy Sets and Systems 224 (2013) 2335

[13] D. Jočić, I. Štajner-Papuga, Some implications of the restricted distributivity of aggregation operators with absorbing elements for utility theory, Fuzzy Sets and Systems 291 (2016) 54-65

[14] D. Jočić, I. Štajner-Papuga, Distributivity and conditional distributivity for $T$-uninorms, Information Sciences 424 (2018) 91-103

[15] E. P. Klement, R. Mesiar, E. Pap, Triangular Norms, Kluwer Academic Publishers, Dordrecht, 2000

[16] G. Li, H.-W. Liu, Distributivity and conditional distributivity of uninorm with continuous underlying operators over a continuous t-conorm, Fuzzy Sets and Systems 287 (2016) 154-171

[17] G. Li, H.-W. Liu, Y. Su, On the conditional distributivity of nullnorms over uninorms, Information Sciences 317 (2015) 157-169

[18] M. Mas, G. Mayor, J. Torrens, The distributivity condition for uninorms and t-operators, Fuzzy Sets and Systems 128 (2002) 209-225

[19] M. Mas, G. Mayor, J. Torrens, Corrigendum to "The distributivity condition for uninorms and t-operators" [Fuzzy Sets and Systems 128 (2002), 209-225], Fuzzy Sets and Systems 153 (2005) 297-299

[20] M. Mas, R. Mesiar, M. Monserat, J. Torrens, Aggregation operations with annihilator, Internat. J. Gen. System 34 (2005) 1-22

[21] D. Ruiz, J. Torrens, Distributivity and conditional distributivity of uninorm and a continuous t-conorm. IEEE Transactions on Fuzzy Systems 14 (2) (2006) $180-190$

[22] W. Sander, J. Siedekum, Multiplication, distributivity and fuzzy-integral I, II, III, Kybernetika 41 (2005) 397-422; 469-496; 497-518

[23] Y. Su, H.-W. Liu, D. Ruiz-Aguilera, J. Vicente Riera, J. Torrens, On the distributivity property for uninorms, Fuzzy Sets and Systems 287 (2016) 184-202

[24] Y. Su, W. Zong, H.-W. Liu, On distributivity equations for uninorms over semi-t-operators, Fuzzy Sets and Systems 299 (2016) 41-65

[25] Y. Su, W. Zong, H.-W. Liu, P. Xue, The distributivity equations for semi-toperators over uninorms, Fuzzy Sets and Systems 287 (2016) 172-183

[26] M. Takács, Approximate Reasoning in Fuzzy Systems Based on Pseudoanalysis and Uninorm Residuum, Acta Polytechnica Hungarica 1 (2004) 49-62

[27] R. R. Yager, A. Rybalov, Uninorm aggregation operators, Fuzzy Sets and Systems 80 (1996) 111-120 